\title{
Looking backwards and forward at the same time
}

\author{
Jon Karlsson ${ }^{1} \cdot$ Roland Becker $^{2} \cdot$ Michael Hirschmann $^{3} \cdot$ Volker Musahl $^{4}$
}

Published online: 12 December 2017

(c) European Society of Sports Traumatology, Knee Surgery, Arthroscopy (ESSKA) 2017

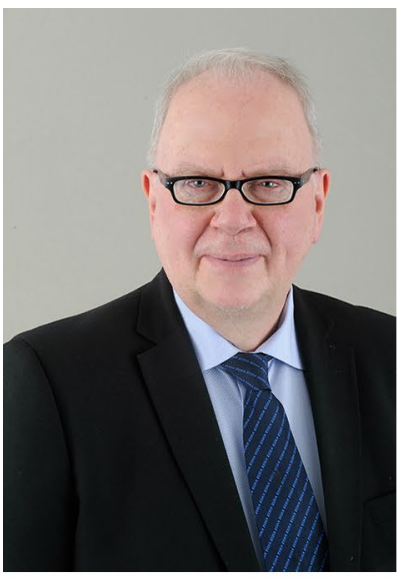

Jon Karlsson

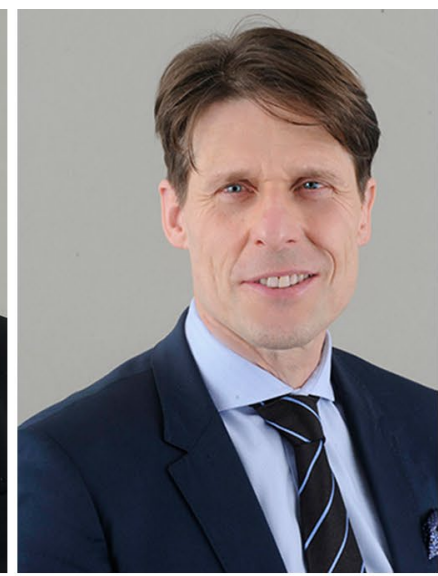

Roland Becker
This year we celebrated the 25th anniversary of the KSSTA journal. Those of us involved in the journal-some of us on a daily basis - are extremely proud of our journal. We have seen it growing and becoming strong. In fact, we can proudly say that it is now one of the leading journals in the world, both in the categories of orthopaedics and sports sciences. KSSTA has evolved and increased in size and content. This has happened year by year. Looking back 10 years, the journal has increased in size from 90 pages per month to 325 pages per month, and at the same time the impact factor

Jon Karlsson

jon.karlsson@telia.com

1 Department of Orthopaedics, Sahlgrenska University Hospital, Mölndal, Sweden

2 Department of Orthopaedic and Traumatology, City Hospital Brandenburg, Hochstrasse 29, 14770 Brandenburg/havel, Germany

3 Department of Orthopaedic Surgery and Traumatology, Kantonsspital Baselland (Bruderholz, Liestal, Laufen), Bruderholz, Switzerland

4 Orthopaedic Surgery and Bioengineering, University of Pittsburgh, UPMC Center for Sports Medicine, 3200 South Water Street, Pittsburgh, PA 15203, USA

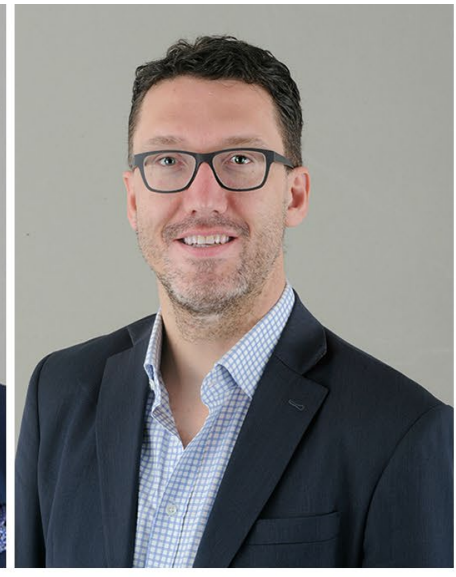

Michael T. Hirschmann

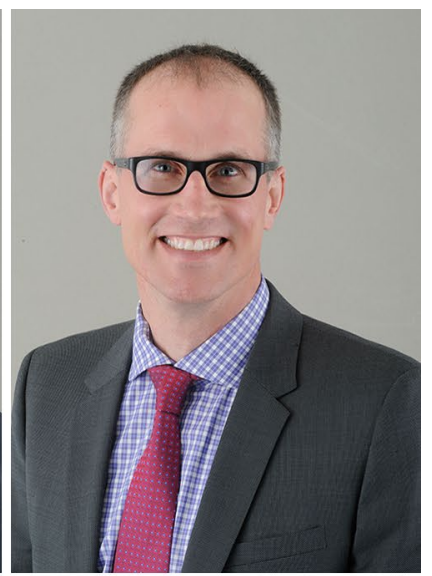

Volker Musahl has increased every year and is now well above 3.0. Again, we strongly feel that we are well established as a " $3+$ journal". But, we aim higher, of course. That can only be done by attracting and publishing the very best papers.

We count on more than 650 reviewers and without their tireless work there would be no journal. The high-quality work by the reviewers is reflected by an increasing number of published papers with high scientific impact. We are immensely grateful to the reviewers doing high-quality work week after week. The only reward for doing good work is more work, as we all know. Thank you reviewers, for your good and timely work. Thank you very much, again.

We, the editors, have discussed a few changes that we hope will lead us forward and make the journal even better. We would like to increase the discussion around the journal in an open and constructive manner. We would like to encourage journal authors and readers to raise their voice and ask critical scientific questions, which may open a constructive atmosphere around the journal. In the same way, we will change the traditional meetings during the ESSKA Congress and make them more interactive and in this manner we can maintain our intention to publish the 
strongest papers. This will also allow us to maintain and improve the impact factor even further.

We have seen a significant increase in the number of published articles. Currently, we publish around 40 papers every month. This means that out of 1,600 submissions, we publish almost 500 papers. But, this also means that we reject more than 1,000 manuscripts every year. This year, our rejection rate will be the highest ever. However, we are thankful that you consider our journal for publication of your scientific work even when we are unable to accept all the submissions. We have noticed an interesting trend that over a 20 -year period the mean number of authors per article has increased from four to almost six; in other words, more and more researchers are involved. We have also noticed a significant increase in the number of Level I and Level II studies. In 1995, the journal published 42 articles, and in 2015 the number had risen to 521 articles. We have published articles in some new categories such as historical papers, often more than one editorial per issue; we try hard to publish controversial editorials, papers, which will be remembered and discussed; we also do our best to follow the trends, for instance anterolateral knee rotatory instability (a full theme issue was published in 2017) and same day knee arthroplasty surgery. However, today we publish fewer experimental studies, as most of them are transferred to our partner, Journal of Experimental Orthopaedics.

We have noticed that the number of submitted systematic reviews and meta-analyses have increased significantly. These studies are able to improve the scientific content and we encourage submission of this type of papers. However, these papers need to be of high scientific quality. In addition, these papers need to add to our current knowledge and be able to change patient care.

There will be no change in the editorial team and we are excited to announce that the editorial team will remain the same as last year. At the ESSKA Congress in Glasgow, we will announce the reviewers of the year 2017. To tell you the truth, we are having big difficulty in selecting only three reviewers, due to the high quality of your work.

Again, we also would like to thank Springer Verlag, especially Gabriele Schröder for a fruitful collaboration over the years. A big thank you also goes to the ESSKA Board and the ESSKA office. Thank you, in particular, to Romain Seil and Zhanna Kovalchuk for the constructive collaboration. Last, but not least, we would like to express our gratitude to our editorial office team, under the leadership of Runeeta Rai: you deserve the biggest "thank you"! 
Elmar Herbst

Reha Tandogan

Antonia Chen

Paulo Araujo

Riccardo Compagnoni

Jon Drogset

Sufian Ahmad

Nobuo Adachi

Andrea Achtnich

Gunnar Knutsen

Takeshi Muneta

Takanori Iriuchishima

Yuichi Hoshino

Mark Hutchinson

Paolo Bulgheroni

Patrick Weber

Daniel Guenther

Andreas Imhoff

Thomas Bauer

Gunter Spahn

Kristín Briem

Hanna Bjørnsson Hallgren

Max Ettinger

Reinoud W. Brouwer

Mo Saffarini

Nicolas Pujol

Carola van Eck

Robert LaPrade

Bogdan Ambrozic

Baris Kocaoglu

Miguel Ruiz Iban

Ulrich Bosch

Albert Lin

Christoph Becher

Giuseppe Milano

Vincenzo Condello

Matthias Brockmeyer

Martijn Brinkman

Katarina Nilsson-Helander

Turgay Efe

Olaf Lorbach

Lucio Ernlund

Mike Baums

Karl Eriksson

Elizabeth Arendt

Alberto Ventura

Oliver Kessler

Gonzalo Samitier

Giulio Maria Marcheggiani Muccioli

Wolfgang Nebelung

Claudio Rosso

Martin Clauss

Klaus Draenert
Truls Straume Næsheim

Christian Stärke

Knut Beitzel

Mattias Ahldén

Tim Spalding

Patrick Orth

Jack Andrish

Alfredo Schiavone Panni

Pedro Gomez

Giuseppe Filardo

Christoph Kittl

Felix Dyrna

Matthieu Ollivier

Onur Bilge

Tommaso Roberti di Sarsina

Stefan Mogos

Alberto Grassi

Sverre Löken

Henrik Aagaard

Lieven De Wilde

Jens Agneskirchner

Martin Lind

David Dejour

Thilo Patzer

Mustafa Karahan

Geert Pagenstert

Philip Roessler

Michael Iosifidis

Nicola Lopomo

Kanto Nagai

Robert Brophy

Masataka Deie

Kamal Deep

Tommaso Bonanzinga

Octav Russu

Michael Krogsgaard

Nicolaas Budhiparama

Simon Donell

Khaled Meknas

Henrik Behrend

Lars Ejerhed

Defne Kaya

Daisuke Araki

Inge Skråmm

Bruno Violante

Fabio Conteduca

Michael Liebensteiner

Joerg Luetzner

Nanne Kort

Anne Kathrine Sørensen

Carl Haasper

Marc Tompkins

Alfred Hochrein 
Karin Grävare Silbernagel

Hélder Pereira

Luigi Pederzini

Elvire Servien

Thomas Muellner

Eva Zeisig

Michael Hantes

Peter Balcarek

Roland Thomee

John Nyland

Mike Carmont

Scott Rodeo

Andreas Scharpf

Bent Lund

Michael Frink

Craig Mauro

Anders Stålman

Gustavo Arliani

Björn Barenius

Brian Devitt

Sven Lichtenberg

Jeffrey Macalena

Frank Martetschläger

Matej Drobnic

Oliver Schindler

Chadwick Prodromos

Mirco Herbort

Jason Koh

Ioannis Kostogiannis

Filippo Randelli

Jae-Heon Jeong

Einar Andreas Sivertsen

Thomas Tischer

Ronald van Heerwaarden

Mari Lundberg

Philippe Massin

Paolo Arrigoni

Alli Gokeler

Kevin Plancher

Jeffrey Abrams

Etienne Belzile

Annelie Brorsson

Andreas Halder

William Walsh

Keith Lawhorn

Bengt Eriksson

James Cowan

Massimo Berruto

Seth Sherman

Manfred Nelitz

Enrique Gomez-Barrena

Martin Pietsch

Fritz Thorey
Enric Castellet

Remco van Wensen

Corrado Bait

Christian Fink

Joerg Jerosch

Sebastien Lustig

Caroline Mouton

Kristian Samuelsson

Christophe Hulet

Eduard Alentorn-Geli

Hermann-Otto Mayr

Mahmut Doral

Michel P. J. van den Bekerom

Carlo Camathias

Rudi Bitsch

Cheng-Kung Cheng

Eivind Inderhaug

Erkal Bilgic

Petra Heesterbeek

Norimasa Nakamura

Philippe Beaufils

Thomas De Bo

Dennis Liem

Björn Salomonsson

Tomoyuki Matsumoto

Nicklas Olsson

Alan Getgood

Antonio Cartucho

Adad Baranto

Sandro Kohl

Rover Krips

Philippe Wilmes

Tobias Dietrich

Thomas Gill

Koen Defoort

Jan Harald Røtterud

Markus Waldén

Christian Gerhardt

Anna Hirschmann

Pablo Gelber

Daniel Hensler

Maximilian Petri

Ofer Levy

Inigo Martinez

Marco De Gori

Darren de SA

Sjoerd Stufkens

Bjoern Rath

Guillaume Demey

Robert Steensen

Tim Boymans

Frantzeska Zampeli

Stephen Lyman 
Jeremy Burnham

Joerg Harrer

Lior Laver

Alastair Younger

Aidin Eslam Pour

Kristoffer Barfod

Siegfried Hofmann

Magnus Forssblad

Henning Madry

Julian Feller

Joanna Kvist

Burt Klos

Bruce Levy

Arsi Harilainen

Roberto Rossi

Niek van Dijk

Romain Seil

Christopher Pearce

Nicola Maffulli

Kazunori Yasuda

Patrick Sadoghi

Johannes Beckmann

Mehmet Erdil

Danny Whelan

Rahul Patel

Denise Eygendaal

Tiberiu Bataga

Stefan Grote

Johann Henckel

Filippo Familiari

Antonio Marmotti

Ingo Banke

Juan Monllau

Clemens Gwinner

Frank Petrigliano

Giuseppe Peretti

Roberto Seijas

Duncan Meuffels

Koichi Kobayashi

Alberto Gobbi

Francisco Forriol

Konstantinos Makridis

Paul Ackermann

Fredrik Einarsson

Lars Blønd

Ricardo de Casas

Sabine Lippacher

John Gliatis

Joon Ho Wang

Matthias Feucht

Martin Hägglund

Stephen Howell

Emmanuel Audenaert
Bertrand Sonnery-Cottet

Andreas Gomoll

Vicente Sanchis-Alfonso

Leendert Blankevoort

Mattia Loppini

Ji-Hoon Bae

Elizaveta Kon

Hideyuki Koga

Elias Tsepis

Vladimir Martinek

Mikel Reilingh

Sebastien Parratte

Jack Lysholm

Jesper Augustsson

Rob Janssen

Pisit Lertwanich

Paolo Adravanti

Olivier van der Meijden

Lars Weidenhielm

Michel Bonnin

Jonas Thorlund

Stefan Marlovits

Takumi Nakagawa

Håvard Moksnes

Nathan Endres

Eric Hamrin Senorski

Frederic Picard

Simon Thompson

Yogesh Kolwadkar

Marco Valoroso

Richard napier

Bernhard Waibl

MaCalus Hogan

Christian Owesen

Radu Fleaca

Jack Farr

Holly Silvers

David Pokorny

Shwan Khoschnau

Christian Baumgart

Rupesh Tarwala

Johannes Wiegerinck

Aaron Krych

Edoardo Franceschetti

Verena Schreiber

Mark Glazebrook

Kelley Fitzgerald

Kilian Wegmann

JoannA Stephen

Werner Krutsch

Philipp Henle

Sandro Giannini

Ewould Van Arkel 
Stavros Ristanis

Matthew Milewski

Srino Bharam

Stéphane Guillo

Tahsin Beyzadeoglu
Peter Myers

Rayan Baalbaki

Karol Zyto

Rik Kundra 